\title{
Mortality among people hospitalised with covid-19 in Switzerland: a
}

\section{nationwide population-based analysis}

Nanina Anderegg ${ }^{\mathrm{a}, \mathrm{b}}$ (0000-0002-8238-8832), Radoslaw Panczak (0000-0001-5141-683X), Matthias Egger $^{a, c, d}$ (0000-0001-7462-5132), Nicola Low ${ }^{a}$ (0000-0003-4817-8986), Julien Riou ${ }^{a, b}$ (0000-0002$3808-2852)$

anstitute of Social and Preventive Medicine, University of Bern, Switzerland

${ }^{b}$ Federal Office of Public Health, Bern, Switzerland

${ }^{\mathrm{c}}$ Centre for Infectious Disease Epidemiology and Research, Faculty of Health Sciences, University of Cape Town, Cape Town, South Africa

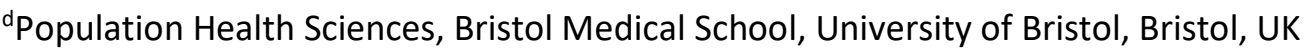

\section{Correspondence to:}

Nanina Anderegg PhD

Institute of Social and Preventive Medicine

University of Bern, Bern, Switzerland

nanina.anderegg@ispm.unibe.ch

Word count: abstract 338, main text 3686, 1 table, 5 figures, 6 supplementary figures, 1 supplementary table, 1 supplementary text, 43 references. 


\section{Abstract}

Objectives: To investigate mortality among people hospitalised with covid-19 in Switzerland according to epidemic wave, age, sex, comorbid conditions and intensive care unit (ICU) occupancy.

Design: Population-based, national study.

Setting: Mandatory surveillance reports from all hospitals in Switzerland.

Participants: All 22,648 people who tested positive for SARS-CoV-2 infection and were hospitalised between February 24, 2020 and March 01, 2021 in Switzerland with complete information about age, sex, and comorbidities.

Main outcome measures: Survival after positive SARS-CoV-2 test among people hospitalised with covid-19 by epidemic wave, age, sex, comorbid conditions and ICU occupancy, expressed as adjusted hazard ratios (aHR) of death and probability of survival over time and at 40 days, all with $95 \%$ credible intervals (Crl).

Results: Of 22,648 people hospitalised with covid-19, 4,785 (21.1\%) died. Bayesian survival models adjusted for age, sex, and the presence of comorbidity showed that survival was lower during the first epidemic wave than the second (standardised predicted survival probability at 40 days $76.1 \%$ versus 80.5\%; aHR of death $1.38,95 \% \mathrm{Crl} 1.28$ to 1.48 ). During the second epidemic wave, occupancy among all available ICU beds (certified beds and add-on beds) in Switzerland varied between $51.7 \%$ and $78.8 \%$. Modelling the association between survival and ICU occupancy with restricted cubic splines indicated stable survival when ICU occupancy was below $70 \%$, but worse survival when ICU occupancy exceeded $70 \%$. This threshold of $70 \%$ occupancy among total available ICU beds corresponded to around $85 \%$ occupancy among certified beds. Survival was decreased for men, older people, and patients with comorbid conditions. Comorbid conditions reduced survival more in younger people than in older people. As single comorbid condition, hypertension was not associated with poorer survival, but appeared to increase the risk of death in combination with a cardiovascular disease.

Conclusion: Survival after hospitalisation with covid-19 has improved over time, consistent with improved management of severe covid-19. The decreased survival starting at approximately $70 \%$ ICU occupancy in Switzerland supports the need to introduce measures for prevention and control of SARSCoV-2 transmission in the population far before ICUs are full. 


\section{Introduction}

More than five million people have died from coronavirus disease 19 (covid-19) and almost 250 million confirmed cases of severe acute respiratory syndrome coronavirus 2 (SARS-CoV-2), the cause of covid19 , have been reported worldwide as of November 08, 2021 [1-3]. The risk of death from SARS-CoV-2 infection increases with age and is higher for men than women [4-12]. In addition, people with preexisting comorbid conditions, including cancer, cardiovascular disease and chronic respiratory disease are at higher risk of severe covid-19 or death than people without these conditions $[7,8,13-18]$. The interplay between different comorbid conditions and their associated risk of death for different ages or genders is not yet fully understood.

In many countries, a first wave of SARS-CoV-2 infection in the first half of 2020 was controlled by strict lockdown measures but a second larger wave followed the delayed re-introduction of control measures in autumn 2020. Switzerland, a high-income country, experienced the highest levels of excess mortality since the 1918 influenza pandemic, with a larger death toll during the second compared to the first epidemic wave [19]. Nevertheless, outcomes in patients hospitalised with covid19 might have improved over time, as seen in other countries [20].

As in many other countries, the number of free intensive care unit (ICU) beds has become an indicator of the need to introduce or intensify public health measures to control SARS-CoV-2 transmission in Switzerland. Elsewhere, high ICU occupancy during peaks of the epidemic led to worse outcomes in hospitalised covid-19 patients, possibly due to operational pressure [21,22]. It is unknown to what extend this applies to Switzerland. In addition, it remains unclear if outcomes in hospitalised covid-19 patients deteriorate linearly with lower ICU occupancy, or if there is a point of occupancy, beyond which deterioration increases.

The objectives of this study were to examine differences in survival between the first and second epidemic wave in Switzerland, the influence of level of ICU occupancy on survival, and the interplay between different comorbid conditions and other risk factors. 


\section{Methods}

\section{Setting and data}

We analysed data for the whole of Switzerland from national routine surveillance of SARS-CoV-2 infections, conducted by the Swiss Federal Office of Public Health (FOPH). The surveillance system includes all people who tested positive for SARS-CoV-2 infection in Switzerland since February 24, 2020, when the first case of covid-19 was diagnosed. Laboratories report the date of the positive test and the age and sex of the person. For patients hospitalised, medical personnel report the date of hospitalisation and information about specific comorbid conditions. For patients who die, medical personnel report the date of death and presence of comorbid conditions. For the present analysis, we included all hospitalised covid-19 patients with a positive test for SARS-CoV-2 infection between February 24, 2020, and March 01, 2021, and who had complete information about age, sex and comorbid conditions. The database was closed on May 20, 2021, so that all patients were followed up for at least 80 days after the positive test.

The Swiss Armed Forces delivered daily aggregated data from all hospitals in Switzerland about the total number of available ICU beds and the number of occupied beds since March 14, 2020. The total occupied includes both covid-19 and non-covid-19 patients. The total available consists of a relatively stable number of ICU beds over the whole period ('certified ICU beds') and a variable number of additional ICU beds, which have been mobilised, depending on the state of the epidemic in the country. These are called 'add-on' ICU beds.

\section{Outcomes}

The outcome was survival after a positive test for SARS-CoV-2 infection in hospitalised patients. We measured follow-up time from the positive SARS-CoV-2 test to the date of death for patients who died by May 20, 2021. We assumed that deaths occurring more than 80 days after infection are not attributable to covid-19. The date of hospital discharge could not be retrieved, as the surveillance system does not require a follow-up report after hospital admission. We therefore censored follow-up time at 80 days after the positive test for people who died more than 80 days after a positive test and for all people alive at database closure.

\section{Explanatory variables}

Explanatory variables were: the epidemic wave, sex and age, comorbidities and ICU occupancy. We defined the first epidemic wave as the period between February 24, 2020, and June 5, 2020; the second wave covered the period between June 6, 2020, and March 01, 2021. The date separating the two waves was based on the nadir of the case counts between the two waves. We defined daily ICU 
occupancy as the proportion of occupied ICU beds (covid-19 and non-covid-19) on a given day divided by the total number of available ICU beds (certified and add-on beds) on the same day. Age was grouped into eight categories, 0-29, 30-39, 40-49, 50-59, 60-69, 70-79, 80-89, and $\geq 90$ years. We included all comorbid conditions that were asked for in the surveillance form. These were: cancer, cardiovascular disease, chronic respiratory disease, diabetes, hypertension, immunosuppression, obesity, chronic kidney disease, "other" comorbidity and no comorbidity. Obesity and chronic kidney disease were added to the surveillance forms from April 16, 2020.

\section{Statistical analyses}

We modelled the hazard of death by fitting parametric survival models in a Bayesian framework. For all survival models in this study, we used cubic M-splines to model baseline hazards and assumed weakly informative priors for the model parameters (Supplementary Text S1)[23]. To compare survival between the two epidemic waves, we used data for the whole study period and fitted a survival model, including the covariates epidemic wave, age, sex, and a binary variable indicating any or no recorded comorbid condition. We also included all two-way interactions between covariates age, sex and comorbidity status. In an additional analysis, we fitted the same model, but included the number of comorbid conditions reported $(0,1,2$ or 3 or more). We used these results to build models studying mortality by ICU occupancy and by type of comorbid condition during the second wave, because data on ICU occupancy, obesity and chronic kidney disease only started during the first wave (Supplementary Text S1).

For ICU occupancy, we modelled the association with mortality using restricted cubic splines with three knots (at the $10 \%, 50 \%$, and $90 \%$ percentiles of the ICU occupancy distribution in our data). We adjusted the model for age, sex, and comorbidity status. We also included the two-way interaction between age and comorbidity status, for which the first model showed statistical evidence. To investigate survival by type of comorbidity, we included the covariates age and sex, as well as the different comorbidity types. We allowed for two-way interactions between the different comorbidity types themselves, between age and the comorbidity types, and between sex and the comorbidity types (Supplementary Text S1).

We report estimates of adjusted hazard ratios (aHR) for death, predicted survival curves over time (i.e. predicted survival probability at day 0 to day 80 after the positive test), and - as a specific example the predicted survival probability at day 40 after the positive test, all with $95 \%$ credible intervals ( $\mathrm{Crl}$ ). We also report standardised survival probabilities by specific covariates (epidemic wave and ICU occupancy). We standardised by predicting individual survival probabilities for the whole population 
at all levels of the specific covariate(s), and averaging predictions in each of the posterior samples. All analyses were done using R (version 4.0.4) with package rstanarm [23].

Patient and public involvement

As this study uses anonymized, routinely collected surveillance data, it was not feasible to involve study participants or the public in our research's design, conduct, methodology, or dissemination plans. 


\section{Results}

\section{Descriptive statistics}

Overall, there were 559,117 confirmed covid-19 cases between February 24, 2020 and March 01, 2021, of whom 24,515 (4.4\%) were hospitalised (Figure 1). Of all people with confirmed covid-19, 9,711 (17\%) had died by May 20, 2021. Of patients hospitalised, 4,847 (19.8\%) had died, covering $49.9 \%$ of the total reported deaths. People who died outside of hospital include residents of retirement and nursing homes, but information about place of death was incomplete. People older than 90 years accounted for $42.1 \%(1,949 / 4,630)$ of deaths outside hospital and $15.6 \%(746 / 4,785)$ of deaths in hospitalised patients (Supplementary Table S1).

We included 22,648/24,515 (92.4\%) patients hospitalised who had complete covariate information in our analyses ( $\underline{\text { Supplementary Figure S1)}}$. Of all included patients, 4,785 (21.1\%) had died (Table 1). The median (interquartile range [IQR]) age of patients was 74 (62 to 83 ) years and most were male $(13,093$, $57.8 \%)$. Most patients had at least one comorbid condition (19,865, 87.7\%). More people with SARSCoV-2 were hospitalised in the second epidemic wave $(18,952)$ than the first $(3,696)$, but the proportion of people who died among those hospitalised was higher in the first $(24.3 \%)$ than the second wave (20.5\%). Sex and age distributions were similar in the two epidemic waves, as was the presence of specific comorbidities (except for obesity and chronic kidney disease, which were probably mostly recorded as "other" comorbidity in the first wave). During the second wave, the total number of available ICU beds ranged between 963 and 1,143, consisting of 781 to 861 certified beds and 135 to 296 add-on beds (Supplementary Figure S2). ICU occupancy among all available beds ranged between $51.7 \%$ and $78.8 \%$. In periods with low ICU occupancy ( $1^{\text {st }}$ decile, $<65.7 \%$ occupancy) 327 of $1,903(17.2 \%)$ hospitalised covid-19 patients died, while in periods with high ICU occupancy $\left(10^{\text {th }}\right.$ decile, $>76.9 \%$ occupancy) 431 of 1,890 (22.8\%) patients died (Table 1 ).

\section{Survival by epidemic wave}

The baseline risk of death increased sharply after the positive SARS-CoV-2 test, peaked at around 4.5 days and then decreased again, approaching zero at around day 70 (Supplementary Figure S3 ). There was a substantial increase in the hazard of death during the first epidemic wave compared with the second wave, after adjusting for age, sex, and the presence of any comorbid condition (aHR 1.38, 95\% Crl 1.28 to 1.48). The standardised predicted probability of survival at 40 days was $76.1 \%$ (95\% Crl 75.1 to $77.2 \%$ ) during the first and $80.5 \%$ (95\% $\mathrm{Crl} 80.0$ to $81.1 \%$ ) during the second epidemic wave (Supplementary Figure S3). Mortality also increased with male sex, older age and with the presence of any comorbidity (Figure 2). There was evidence for an interaction between comorbidity status and age; except for the age group 0-29 where very few deaths occurred, having any comorbidity increased the hazard of death more at younger than at older ages (igure 3 ). For example in males, the aHR of death 
with comorbidity compared with no comorbidity was 3.94 (95\% $\mathrm{Crl} 2.47$ to 6.64 ) for ages $60-69$ and only 0.97 (95\% Crl 0.55 to 1.90) for ages above 90 . There was no evidence of an interaction between sex and comorbidity status (Figure 3). There was also little evidence of an interaction between sex and age; the difference in mortality by sex in the age groups 0-49 years was unclear owing to small sample sizes, but for patients aged above 50 , there was a consistent increase in the hazard of death by around 1.6 to 1.7 in males compared with females (Figure 3 ). The additional analysis, including the number of comorbidities instead of the comorbidity status, showed that mortality generally increased with the number of reported comorbidities (Supplementary Figure S4). Again, there was a substantial interaction between comorbidity and age group.

\section{Survival by ICU occupancy}

The hazard of death was relatively stable up to around $70 \%$ ICU occupancy and started to increase afterwards (Supplementary Figure S5). Standardised predicted survival at 40 days was stable up to $70 \%$ ICU occupancy, but decreased at higher levels of occupancy (Figure 4). For example, estimated standardised survival at 40 days was similar at 50\% ICU occupancy (81.5\%; $95 \% \mathrm{Crl} 76.3$ to $86.2 \%$ ) and $70 \%$ ICU occupancy (81.0\%; $95 \% \mathrm{Crl} 80.0$ to $81.9 \%$ ). Survival at 40 days started to decrease from this point on, reaching $78.2 \%$ (95\% $\mathrm{Crl} 76.1$ to $80.1 \%$ ) at $80 \%$ ICU occupancy. Focusing on periods that experienced an ICU occupancy exceeding $70 \%$, these results correspond to an estimated number of 137 (95\% Crl 27 to 242 ) deaths - or an estimated proportion of $4.80 \%$ (95\% Crl 0.94 to $8.29 \%$ ) of total deaths during these periods - that could theoretically have been averted if ICU occupancy had stayed below $70 \%$ during these periods. During the second epidemic wave, the threshold of $70 \%$ ICU occupancy among all available beds corresponded to approximately $85 \%$ occupancy among certified beds (Supplementary Figure S2). Associations between mortality and covariates sex, age, and comorbidity status obtained were very similar to those observed with the model comparing the epidemic waves.

\section{Survival by comorbidity type}

In patients $<40$ years old we found insufficient and inconclusive evidence for any of the associations between single comorbid conditions and mortality, as the small number of deaths in these age groups lead to noisy estimates and wide credibility intervals (Figure 5). In patients aged 40 years or older with a single comorbid condition, the strongest association with mortality was cancer. For patients aged 5089 years old, chronic respiratory disease, chronic kidney disease, cardiovascular disease and immunosuppression were also often associated with higher mortality. There was little evidence for worse survival in patients with hypertension or obesity as the only comorbid condition. The patterns of association were similar for males and females. Looking at two-way interactions between comorbidity types, we found that having both hypertension and cardiovascular disease was more 
strongly associated with death than the sum of the individual effects of both (Supplementary Figure S6). This suggests a synergistic effect of these two conditions. Other potential synergies include obesity and diabetes, immunosuppression and chronic kidney disease, or obesity and hypertension. We also found some indication that the combination of obesity and cancer or immunosuppression, or diabetes and chronic respiratory disease were less strongly associated with mortality than the sum of the individual conditions (antagonist effect). However, for most of these combinations, effect sizes were small and credible interval overlapped 1 - indicating weak evidence towards an interaction. 


\section{Discussion}

\section{Principal findings}

This nationwide analysis of almost 23,000 people who were hospitalised with covid-19 in Switzerland found that mortality was around $40 \%$ higher in the first than in the second epidemic wave. We found that survival amongst patients hospitalised with covid-19 started to decrease when ICU occupancy was around $70 \%$ or more. Our study confirmed the higher risk of death in men than in women and in older than in younger people. At younger ages, having any comorbid condition was more strongly associated with poor survival than at older ages. Cancer, chronic respiratory disease, chronic kidney disease, and cardiovascular disease were most strongly associated with poorer survival. We found little evidence for poorer survival in patients with hypertension as single comorbid condition.

\section{Strengths and weaknesses of this study}

Our study uses data from mandatory surveillance for the whole of Switzerland and includes around $92 \%$ of all people with laboratory-confirmed covid-19 who were admitted to any hospital during the first two waves of the epidemic. The use of Bayesian survival models allowed us to fit complex models that included many interactions between variables. For example, we could examine both single comorbid conditions and the interplay between comorbidities.

There are weaknesses in the study that result from the data collected by the national surveillance systems. First, information about obesity and chronic kidney disease as comorbid conditions was only included in mid-April 2020, and data about numbers of ICU beds were only collected from mid-March 2020, so we restricted analyses about ICU occupancy and types of comorbidities to the second wave. Given the improvement in survival over time, we believe that these more up-to-date analyses reflect current clinical management of covid-19. Second, a substantial proportion of covid-19 deaths in older people occurred without hospitalisation and were not included in analyses. It is likely that these deaths occurred mainly in retirement and long-term care institutions, but information about the place of death was incomplete. Our results may not apply to the population of older adults who died without hospitalisation. Third, our study includes the period when the SARS-CoV-2 alpha variant of concern was increasing rapidly in Switzerland, but ended before the delta variant became established [24]. These variants of concern contain mutations that confer increased pathogenicity, compared with the wild type SARS-CoV-2, which dominated until January 2021 [25]. Our findings might therefore underestimate the numbers of people admitted to hospital, and dying from covid-19 since mid-2021, but we believe that the patterns of mortality according to sex, age and type of comorbidity have not been affected. 


\section{Comparison with other studies}

Our study adds evidence about the association between survival with covid-19 and ICU occupancy. Higher mortality during periods of high ICU occupancy has been shown [21,22]. In Belgium, mortality in patients admitted to the ICU was 42\% higher and in England the odds of death were 1.1 times higher in periods of high ICU occupancy. The studies categorised ICU occupancy as a binary or ternary characteristic ("ICU overflow" versus "no ICU overflow" and "0-45\% occupancy" versus "45-85\% occupancy" versus ">85\% occupancy"). The advantage of our analysis is that we modelled the association between survival and ICU occupancy using the flexibility of splines and found a threshold, at around $70 \%$ overall occupancy, after which survival decreases. Whilst this threshold might be specific to Switzerland and to the second epidemic wave, the methodological approach can be applied more widely.

The increased hazard of death in older ages and in men is in line with results from other studies [912]. Our study brings additional knowledge about the association between the presence of comorbidities and increased mortality, which has been shown in other studies $[7,8,14-18]$. We showed that having any comorbidity increased mortality more at younger than at older ages, and that while there are substantial interactions between unique comorbidity types and age, there is no difference of comorbidity effects by sex. We did not find an increase in mortality in patients with obesity as single comorbid condition, which is in contrast to findings from other studies [26]. Our study might underestimate the association between obesity and mortality due to underreporting of obesity: Even though we did restrict analyses to a period where the notification form included obesity as comorbid condition, the new version of the form might not have been adopted immediately by all hospitals. We also did not find an increase in mortality for patients suffering from hypertension alone, which is in line with other studies from Switzerland [4,5]. In other studies summarised in systematic reviews, hypertension was associated with increase mortality, but these studies did not report on whether hypertension was a sole condition [27-29]. We found that hypertension appeared to increase the risk of death when associated with a cardiovascular disease. This may indicate that as long as the hypertension of patients is under control it is not associated with worse survival, however if a cardiovascular disease arises as a consequence of an uncontrolled hypertension, the risk of death increases. We found other potential interactions between comorbidities in both directions but many findings were based on small numbers of events and require additional investigation. To our knowledge, none of the previous studies included interactions between the different comorbidities, so that they are only able to estimate averaged effects of the individual comorbidities. 


\section{Interpretation and implications}

The better survival during the second wave of covid-19 in Switzerland, compared with the first, is consistent with improvements in the clinical management of people hospitalised with covid-19. These improvements might reflect both increased clinical experience and specific treatments. For example, the use of systemic corticosteroids in patients with severe and critical covid-19 increased after the publication of results from clinical trials and a recommendation from the World Health Organization in September 2020 [30-38]. The decrease in survival starting at approximately $70 \%$ overall ICU occupancy shows a potential impact of operational pressure on mortality in patients hospitalised with covid-19. Estimated absolute differences in survival were small with an only $3.3 \%$ difference in survival on average between lowest and highest ICU occupancy. Nevertheless, this corresponded to an estimated 137 deaths or $4.8 \%$ of all deaths in periods with ICU occupancy $>70 \%$ that could have been averted if ICU occupancy had stayed below $70 \%$. We looked at occupancy among all available certified and add-on beds. During the second epidemic wave, the threshold at $70 \%$ overall ICU occupancy corresponded to approximately $85 \%$ occupancy among certified ICU beds. As ICU are organised differently in most countries our results are not generalizable to other countries. In addition, if provision of add-on ICU beds (or the number of certified beds in use) changes within Switzerland in the future, results might not be applicable to future epidemic waves in the country. Nevertheless, our results suggest the need for prevention and control of SARS-CoV-2 transmission in the population far before ICUs are full.

Our detailed results about the risk of death for separate comorbidities and combinations of comorbidities by age and sex can further help clinicians to better evaluate the prognosis of their patients. For instance, some single comorbid conditions and combinations of conditions appear as particularly risky, which could have significant impact on patient management. The increased hazard of death in men could not be explained by any interactions with comorbidities or age. The reason for the higher mortality in men might be due to residual confounding by an uneven distribution of other risk factors (e.g. smoking, unhealthy diet, less physical activity, socio-economic position), which were not collected and could thus not be adjusted for in analyses [39-42]. Males might also be admitted to the hospital at a more advanced stage of the disease [9]. Lastly, there might be a biological explanation, for example a different immune response in females and males $[41,43]$.

\section{Conclusion}

Survival of hospitalised covid-19 patients has increased over time in Switzerland, possibly due to gained knowledge in treating SARS-CoV-2 infection. Survival was relatively stable until an ICU occupancy of approximately $70 \%$ from where it started to decrease. This indicates operational pressure far before 
ICUs are full and triage becomes necessary. This calls for caution when concluding that the epidemic is under control as long as there is still ICU capacity available. 


\section{SUMMARY BOX}

\section{What is already known on the topic}

- Studies have shown a higher risk of death from SARS-CoV-2 infection for increasing age, for men, and for people with pre-existing comorbidities. The interplay between different comorbid conditions and their associated risk of death for different ages or genders is not yet fully understood.

- A higher mortality for patients admitted to the ICU during periods of high occupancy was shown in two studies from Belgium and the UK. It remains unclear if this applies to Switzerland as well and if outcomes in hospitalised covid-19 patients deteriorate linearly with lower ICU occupancy, or if there is a point of occupancy, beyond which deterioration increases.

\section{What this study adds}

- Our study suggests stable survival when ICU occupancy was below 70\%, but decreased when occupancy among total available ICU beds in Switzerland exceeded 70\%.

- Our study suggests that having a comorbidity has a stronger detrimental effect on survival in younger compared to older ages. It also shows that for patients aged above 40 years, cancer, chronic kidney disease, cardiovascular disease, immunosuppression and chronic respiratory disease were associated with worst survival among individual comorbidities. Hypertension was not associated with poorer survival as single comorbid condition, but appeared particularly risky when associated with a cardiovascular disease.

\section{Ethics statement}

We did this research using surveillance data according to the Swiss law on communicable diseases (EpG, SR 818.101). No ethical committee approval was required.

\section{Data sharing}

The data are accessible to researchers upon reasonable request for data sharing to the corresponding author. Requests for data need to be approved by the Swiss Federal Office of Public Health.

\section{Acknowledgements}

This study would not have been possible without the extraordinary efforts of the data science team at the Swiss Federal Office of Public Health: Samuel Colin, Jeffrey Keller, Anne Laube, Urs Mayr, Damir Perisa, Katrin Schneider, Moritz Wagner, and Biagio Zaffora. 


\section{Footnotes}

\section{Authorship contribution:}

$N A, R P, M E, N L$, and JR have contributed to conceptualization of the study. JR, NL and ME have supervised the study. NA and JR contributed to the methodology and conducted all analyses including visualization of the results. NA, JR, and NL drafted the original manuscript. All authors contributed to interpretation of the results, and revision and editing of the manuscript. JR and NA are the guarantor. The corresponding author attests that all listed authors meet authorship criteria and that no others meeting the criteria have been omitted.

\section{Copyright/license for publication}

The Corresponding Author has the right to grant on behalf of all authors and does grant on behalf of all authors, a worldwide licence to the Publishers and its licensees in perpetuity, in all forms, formats and media (whether known now or created in the future), to i) publish, reproduce, distribute, display and store the Contribution, ii) translate the Contribution into other languages, create adaptations, reprints, include within collections and create summaries, extracts and/or, abstracts of the Contribution, iii) create any other derivative work(s) based on the Contribution, iv) to exploit all subsidiary rights in the Contribution, v) the inclusion of electronic links from the Contribution to third party material where-ever it may be located; and, vi) licence any third party to do any or all of the above.

\section{Competing risk statement}

No competing interests: All authors have completed the ICMJE uniform disclosure form at www.icmje.org/coi_disclosure.pdf and declare: no support from any organization for the submitted work; no financial relationships with any organizations that might have an interest in the submitted work in the previous three years; no other relationships or activities that could appear to have influenced the submitted work.

\section{Transparency statement}

The corresponding author (the manuscript's guarantor) affirms that the manuscript is an honest, accurate, and transparent account of the study being reported; that no important aspects of the study have been omitted; and that any discrepancies from the study as planned have been explained.

\section{Role of the funding source}

This study was funded by the Swiss Federal Office of Public Health and the Swiss National Science Foundation (189498). NL acknowledges funding from the EU's Horizon 2020 research and innovation programme (project EpiPose, 101003688). The funder of the study had no role in study design, data collection, data analysis, data interpretation, or writing of the report. 


\section{References}

1. Hui DS, I Azhar E, Madani TA, et al. The continuing 2019-nCoV epidemic threat of novel coronaviruses to global health - The latest 2019 novel coronavirus outbreak in Wuhan, China. Int. J. Infect. Dis. 2020; 91:264-266. Available at: http://www.ncbi.nlm.nih.gov/pubmed/31953166.

2. World Health Organization (WHO). WHO Director-General's opening remarks at the mission briefing on COVID-19. [Online]. Available from: https://www.who.int/dg/ speeches/detail/who-director-general-s-opening-remarks-at-the-mission- briefing-on-covid19 [Accessed on 1st March 2020]. 2020: 1. Available at: https://www.who.int/directorgeneral/speeches/detail/who-director-general-s-opening-remarks-at-the-mission-briefing-oncovid-19---16-april-2020.

3. World Health Organization (WHO). WHO Coronavirus (COVID-19) Dashboard. Available at: https://covid19.who.int/. Accessed 11 June 2021.

4. Diebold M, Martinez AE, Adam K-M, et al. Temporal trends of COVID-19 related in-hospital mortality and demographics in Switzerland - a retrospective single centre cohort study. Swiss Med. Wkly. 2021; Available at: https://doi.emh.ch/smw.2021.20572.

5. Maximiano Sousa F, Roelens M, Fricker B, et al. Risk factors for severe outcomes for COVID-19 patients hospitalised in Switzerland during the first pandemic wave, February to August 2020: prospective observational cohort study. Swiss Med. Wkly. 2021; Available at: https://doi.emh.ch/smw.2021.20547.

6. Hauser A, Counotte MJ, Margossian CC, et al. Estimation of SARS-CoV-2 mortality during the early stages of an epidemic: A modeling study in Hubei, China, and six regions in Europe. PLOS Med. 2020; 17:e1003189. Available at: https://dx.plos.org/10.1371/journal.pmed.1003189.

7. Du R-H, Liang L-R, Yang C-Q, et al. Predictors of mortality for patients with COVID-19 pneumonia caused by SARS-CoV-2: a prospective cohort study. Eur. Respir. J. 2020; 55:2000524. Available at: http://erj.ersjournals.com/lookup/doi/10.1183/13993003.005242020.

8. Barek MA, Aziz MA, Islam MS. Impact of age, sex, comorbidities and clinical symptoms on the severity of COVID-19 cases: A meta-analysis with 55 studies and 10014 cases. Heliyon 2020; 6:e05684. Available at: http://www.ncbi.nlm.nih.gov/pubmed/33344791.

9. Peckham H, de Gruijter NM, Raine C, et al. Male sex identified by global COVID-19 metaanalysis as a risk factor for death and ITU admission. Nat. Commun. 2020; 11:6317. Available at: http://www.ncbi.nlm.nih.gov/pubmed/33298944.

10. Salje H, Tran Kiem C, Lefrancq N, et al. Estimating the burden of SARS-CoV-2 in France. Science (80-. ). 2020; 369:208-211. Available at: 
https://www.sciencemag.org/lookup/doi/10.1126/science.abc3517.

11. Gudbjartsson DF, Norddahl GL, Melsted P, et al. Humoral Immune Response to SARS-CoV-2 in Iceland. N. Engl. J. Med. 2020; 383:1724-1734. Available at: http://www.nejm.org/doi/10.1056/NEJMoa2026116.

12. Levin AT, Hanage WP, Owusu-Boaitey N, Cochran KB, Walsh SP, Meyerowitz-Katz G. Assessing the age specificity of infection fatality rates for COVID-19: systematic review, meta-analysis, and public policy implications. Eur. J. Epidemiol. 2020; 35:1123-1138. Available at: http://www.ncbi.nlm.nih.gov/pubmed/33289900.

13. Boulle A, Davies M-A, Hussey H, et al. Risk factors for COVID-19 death in a population cohort study from the Western Cape Province, South Africa. Clin. Infect. Dis. 2020; Available at: http://www.ncbi.nlm.nih.gov/pubmed/32860699.

14. Aveyard P, Gao M, Lindson N, et al. Association between pre-existing respiratory disease and its treatment, and severe COVID-19: a population cohort study. Lancet Respir. Med. 2021; Available at: https://linkinghub.elsevier.com/retrieve/pii/S2213260021000953.

15. Harrison SL, Fazio-Eynullayeva E, Lane DA, Underhill P, Lip GYH. Comorbidities associated with mortality in 31,461 adults with COVID-19 in the United States: A federated electronic medical record analysis. PLoS Med. 2020; 17:e1003321. Available at: http://www.ncbi.nlm.nih.gov/pubmed/32911500.

16. Henry BM, Lippi G. Chronic kidney disease is associated with severe coronavirus disease 2019 (COVID-19) infection. Int. Urol. Nephrol. 2020; 52:1193-1194. Available at: http://www.ncbi.nlm.nih.gov/pubmed/32222883.

17. Sanyaolu A, Okorie C, Marinkovic A, et al. Comorbidity and its Impact on Patients with COVID19. SN Compr. Clin. Med. 2020; :1-8. Available at: http://www.ncbi.nlm.nih.gov/pubmed/32838147.

18. Yang J, Zheng Y, Gou X, et al. Prevalence of comorbidities and its effects in patients infected with SARS-CoV-2: a systematic review and meta-analysis. Int. J. Infect. Dis. 2020; 94:91-95. Available at: http://www.ncbi.nlm.nih.gov/pubmed/32173574.

19. Konstantinoudis G, Cameletti M, Gómez-Rubio V, et al. Regional excess mortality during the 2020 COVID-19 pandemic: a study of five European countries. medRxiv 2021; :2021.10.18.21264686. Available at: http://medrxiv.org/content/early/2021/10/23/2021.10.18.21264686.abstract.

20. Dennis JM, McGovern AP, Vollmer SJ, Mateen BA. Improving Survival of Critical Care Patients With Coronavirus Disease 2019 in England: A National Cohort Study, March to June 2020*. Crit. Care Med. 2021; 49:209-214. Available at: https://journals.Iww.com/10.1097/CCM.0000000000004747. 
21. Taccone FS, Van Goethem N, De Pauw R, et al. The role of organizational characteristics on the outcome of COVID-19 patients admitted to the ICU in Belgium. Lancet Reg. Heal. - Eur. 2021; 2:100019. Available at: https://linkinghub.elsevier.com/retrieve/pii/S2666776220300193.

22. Wilde $H$, Mellan T, Hawryluk I, et al. The association between mechanical ventilator availability and mortality risk in intensive care patients with COVID-19: A national retrospective cohort study. medRxiv 2021; :2021.01.11.21249461. Available at: http://medrxiv.org/content/early/2021/01/19/2021.01.11.21249461.abstract.

23. Brilleman SL, Elci EM, Novik JB, Wolfe R. Bayesian Survival Analysis Using the rstanarm R Package. 2020; Available at: https://arxiv.org/abs/2002.09633. Accessed 12 June 2021.

24. Chen C, Nadeau SA, Yared M, Voinov P, Stadler T. CoV-Spectrum: Analysis of globally shared SARS-CoV-2 data to Identify and Characterize New Variants. 2021;

25. Twohig KA, Nyberg T, Zaidi A, et al. Hospital admission and emergency care attendance risk for SARS-CoV-2 delta (B.1.617.2) compared with alpha (B.1.1.7) variants of concern: a cohort study. Lancet Infect. Dis. 2021; Available at: https://linkinghub.elsevier.com/retrieve/pii/S1473309921004758.

26. Anderson MR, Geleris J, Anderson DR, et al. Body Mass Index and Risk for Intubation or Death in SARS-CoV-2 Infection : A Retrospective Cohort Study. Ann. Intern. Med. 2020; 173:782-790. Available at: http://www.ncbi.nlm.nih.gov/pubmed/32726151.

27. Du Y, Zhou N, Zha W, Lv Y. Hypertension is a clinically important risk factor for critical illness and mortality in COVID-19: A meta-analysis. Nutr. Metab. Cardiovasc. Dis. 2021; 31:745-755. Available at: http://www.ncbi.nlm.nih.gov/pubmed/33549450.

28. Poly TN, Islam MM, Yang HC, et al. Obesity and Mortality Among Patients Diagnosed With COVID-19: A Systematic Review and Meta-Analysis. Front. Med. 2021; 8:620044. Available at: http://www.ncbi.nlm.nih.gov/pubmed/33634150.

29. Barron E, Bakhai C, Kar P, et al. Associations of type 1 and type 2 diabetes with COVID-19related mortality in England: a whole-population study. Lancet Diabetes Endocrinol. 2020; 8:813-822. Available at: https://linkinghub.elsevier.com/retrieve/pii/S2213858720302722.

30. World Health Organization (WHO). Corticosteroids for COVID-19 - Living Guidance. 2020. Available at: https://www.who.int/publications/i/item/WHO-2019-nCoV-Corticosteroids2020.1.

31. The RECOVERY Collaborative Group*. Dexamethasone in Hospitalized Patients with Covid-19. N. Engl. J. Med. 2021; 384:693-704. Available at: http://www.nejm.org/doi/10.1056/NEJMoa2021436.

32. WHO Rapid Evidence Appraisal for COVID-19 Therapies (REACT) Working Group, Sterne JAC, 
Murthy S, et al. Association Between Administration of Systemic Corticosteroids and Mortality Among Critically III Patients With COVID-19: A Meta-analysis. JAMA 2020; 324:1330-1341.

Available at: http://www.ncbi.nlm.nih.gov/pubmed/32876694.

33. Siemieniuk RA, Bartoszko JJ, Ge L, et al. Drug treatments for covid-19: living systematic review and network meta-analysis. BMJ 2020; 370:m2980. Available at: http://www.ncbi.nlm.nih.gov/pubmed/32732190.

34. Tomazini BM, Maia IS, Cavalcanti AB, et al. Effect of Dexamethasone on Days Alive and Ventilator-Free in Patients With Moderate or Severe Acute Respiratory Distress Syndrome and COVID-19: The CoDEX Randomized Clinical Trial. JAMA 2020; 324:1307-1316. Available at: http://www.ncbi.nlm.nih.gov/pubmed/32876695.

35. Dequin P-F, Heming N, Meziani F, et al. Effect of Hydrocortisone on 21-Day Mortality or Respiratory Support Among Critically III Patients With COVID-19: A Randomized Clinical Trial. JAMA 2020; 324:1298-1306. Available at: http://www.ncbi.nlm.nih.gov/pubmed/32876689.

36. Ye Z, Wang Y, Colunga-Lozano LE, et al. Efficacy and safety of corticosteroids in COVID-19 based on evidence for COVID-19, other coronavirus infections, influenza, community-acquired pneumonia and acute respiratory distress syndrome: a systematic review and meta-analysis. CMAJ 2020; 192:E756-E767. Available at: http://www.ncbi.nlm.nih.gov/pubmed/32409522.

37. Corral-Gudino L, Bahamonde A, Arnaiz-Revillas F, et al. Methylprednisolone in adults hospitalized with COVID-19 pneumonia : An open-label randomized trial (GLUCOCOVID). Wien. Klin. Wochenschr. 2021; 133:303-311. Available at: http://www.ncbi.nlm.nih.gov/pubmed/33534047.

38. Angus DC, Derde L, Al-Beidh F, et al. Effect of Hydrocortisone on Mortality and Organ Support in Patients With Severe COVID-19: The REMAP-CAP COVID-19 Corticosteroid Domain Randomized Clinical Trial. JAMA 2020; 324:1317-1329. Available at: http://www.ncbi.nlm.nih.gov/pubmed/32876697.

39. Riou J, Panczak R, Althaus CL, et al. Socioeconomic position and the COVID-19 care cascade from testing to mortality in Switzerland: a population-based analysis. Lancet Public Heal. 2021; 6:e683-e691. Available at: https://linkinghub.elsevier.com/retrieve/pii/S2468266721001602.

40. Yuki K, Fujiogi M, Koutsogiannaki S. COVID-19 pathophysiology: A review. Clin. Immunol. 2020; 215:108427. Available at: https://linkinghub.elsevier.com/retrieve/pii/S152166162030262X.

41. Dehingia N, Raj A. Sex differences in COVID-19 case fatality: do we know enough? Lancet Glob. Heal. 2021; 9:e14-e15. Available at: https://linkinghub.elsevier.com/retrieve/pii/S2214109X20304642. 
42. Jordan RE, Adab P, Cheng KK. Covid-19: risk factors for severe disease and death. BMJ 2020; :m1198. Available at: https://www.bmj.com/lookup/doi/10.1136/bmj.m1198.

43. Pradhan A, Olsson P-E. Sex differences in severity and mortality from COVID-19: are males more vulnerable? Biol. Sex Differ. 2020; 11:53. Available at:

http://www.ncbi.nlm.nih.gov/pubmed/32948238. 
Table 1. Characteristics and mortality of patients hospitalised with a positive SARS-CoV-2 test during the first and second epidemic wave in Switzerland.

\begin{tabular}{|c|c|c|c|c|c|c|}
\hline & \multicolumn{3}{|c|}{$\begin{array}{c}\text { First epidemic wave } \\
\text { February } 24 \text { to June 5, } 2020\end{array}$} & \multicolumn{3}{|c|}{$\begin{array}{c}\text { Second epidemic wave } \\
\text { June 6, } 2020 \text { to March 1, } 2021\end{array}$} \\
\hline & No. of patients & Deaths & $\%$ & No. of patients & Deaths & $\%$ \\
\hline \multicolumn{7}{|l|}{ Total } \\
\hline & 3696 & 897 & $24.3 \%$ & 18952 & 3888 & $20.5 \%$ \\
\hline \multicolumn{7}{|l|}{ Sex } \\
\hline Female & 1456 & 292 & $20.1 \%$ & 8099 & 1381 & $17.1 \%$ \\
\hline Male & 2240 & 605 & $27.0 \%$ & 10853 & 2507 & $23.1 \%$ \\
\hline \multicolumn{7}{|l|}{ Age [years] } \\
\hline Median (IQR) & $73(59-82)$ & \multicolumn{2}{|c|}{$81(74-87)$} & $75(63-83)$ & \multicolumn{2}{|c|}{$82(75-87)$} \\
\hline $0-29$ & 137 & 1 & $0.7 \%$ & 517 & 3 & $0.6 \%$ \\
\hline $30-39$ & 122 & 4 & $3.3 \%$ & 399 & 4 & $1.0 \%$ \\
\hline $40-49$ & 202 & 4 & $2.0 \%$ & 874 & 22 & $2.5 \%$ \\
\hline $50-59$ & 505 & 31 & $6.1 \%$ & 2009 & 98 & $4.9 \%$ \\
\hline $60-69$ & 624 & 94 & $15.1 \%$ & 3274 & 382 & $11.7 \%$ \\
\hline $70-79$ & 920 & 252 & $27.4 \%$ & 4976 & 1107 & $22.2 \%$ \\
\hline $80-89$ & 906 & 371 & $40.9 \%$ & 5360 & 1666 & $31.1 \%$ \\
\hline $90+$ & 280 & 140 & $50.0 \%$ & 1543 & 606 & $39.3 \%$ \\
\hline \multicolumn{7}{|l|}{ Comorbidities } \\
\hline \multicolumn{7}{|l|}{ Presence of any comorbidity } \\
\hline No comorbidity & 495 & 19 & $3.8 \%$ & 2288 & 88 & $3.8 \%$ \\
\hline Any comorbidity & 3201 & 878 & $27.4 \%$ & 16664 & 3800 & $22.8 \%$ \\
\hline \multicolumn{7}{|l|}{ Number of comorbidities } \\
\hline 0 & 495 & 19 & $3.8 \%$ & 2288 & 88 & $3.8 \%$ \\
\hline 1 & 1184 & 149 & $12.6 \%$ & 5253 & 569 & $10.8 \%$ \\
\hline 2 & 1006 & 254 & $25.2 \%$ & 4809 & 901 & $18.7 \%$ \\
\hline $3+$ & 1011 & 475 & $47.0 \%$ & 6602 & 2330 & $35.3 \%$ \\
\hline \multicolumn{7}{|l|}{$\begin{array}{l}\text { Presence of specific } \\
\text { comorbidities }\end{array}$} \\
\hline Cancer & 382 & 182 & $47.6 \%$ & 2203 & 795 & $36.1 \%$ \\
\hline Cardiovascular disease & 1244 & 534 & $42.9 \%$ & 8057 & 2561 & $31.8 \%$ \\
\hline Chronic kidney disease & 90 & 61 & $67.8 \%$ & 3803 & 1437 & $37.8 \%$ \\
\hline Chronic respiratory disease & 578 & 209 & $36.2 \%$ & 3135 & 960 & $30.6 \%$ \\
\hline Diabetes & 844 & 258 & $30.6 \%$ & 4875 & 1274 & $26.1 \%$ \\
\hline Hypertension & 1921 & 602 & $31.3 \%$ & 9781 & 2551 & $26.1 \%$ \\
\hline Immunosuppression & 153 & 63 & $41.2 \%$ & 1036 & 322 & $31.1 \%$ \\
\hline Obesity & 16 & 8 & $50.0 \%$ & 1844 & 430 & $23.3 \%$ \\
\hline Other & 1500 & 481 & $32.1 \%$ & 4657 & 1254 & $26.9 \%$ \\
\hline \multicolumn{7}{|l|}{ ICU occupancy } \\
\hline \multicolumn{7}{|l|}{ Total ICU occupancy } \\
\hline$\leq 65.7^{\&} \%$ & - & - & & 1903 & 327 & $17.2 \%$ \\
\hline $65.8 \%-72.8^{\&} \%$ & - & - & & 7622 & 1521 & $20.0 \%$ \\
\hline $72.9 \%-76.9^{\&} \%$ & - & - & & 7537 & 1609 & $21.3 \%$ \\
\hline$>76.9^{\&} \%$ & - & - & & 1890 & 431 & $22.8 \%$ \\
\hline
\end{tabular}

${ }^{\&} 65.7,72.8$, and $76.9 \%$ correspond to the $10 \%, 50 \%$ and $90 \%$ percentiles of the ICU occupancy distribution in the second epidemic wave. Abbreviations: ICU = intensive care unit

Figures correspond to Counts (Percentage) or Median (interquartile range). 
Figure 1. The SARS-CoV-2 epidemic in Switzerland. Total confirmed cases from February 24, 2020 to March 01,2021 (upper panel), confirmed hospitalised patients (middle panel), and total deaths and deaths in patients hospitalised until May 20, 2021 (lower panel). The dashed vertical lines correspond to the end of the first epidemic wave.
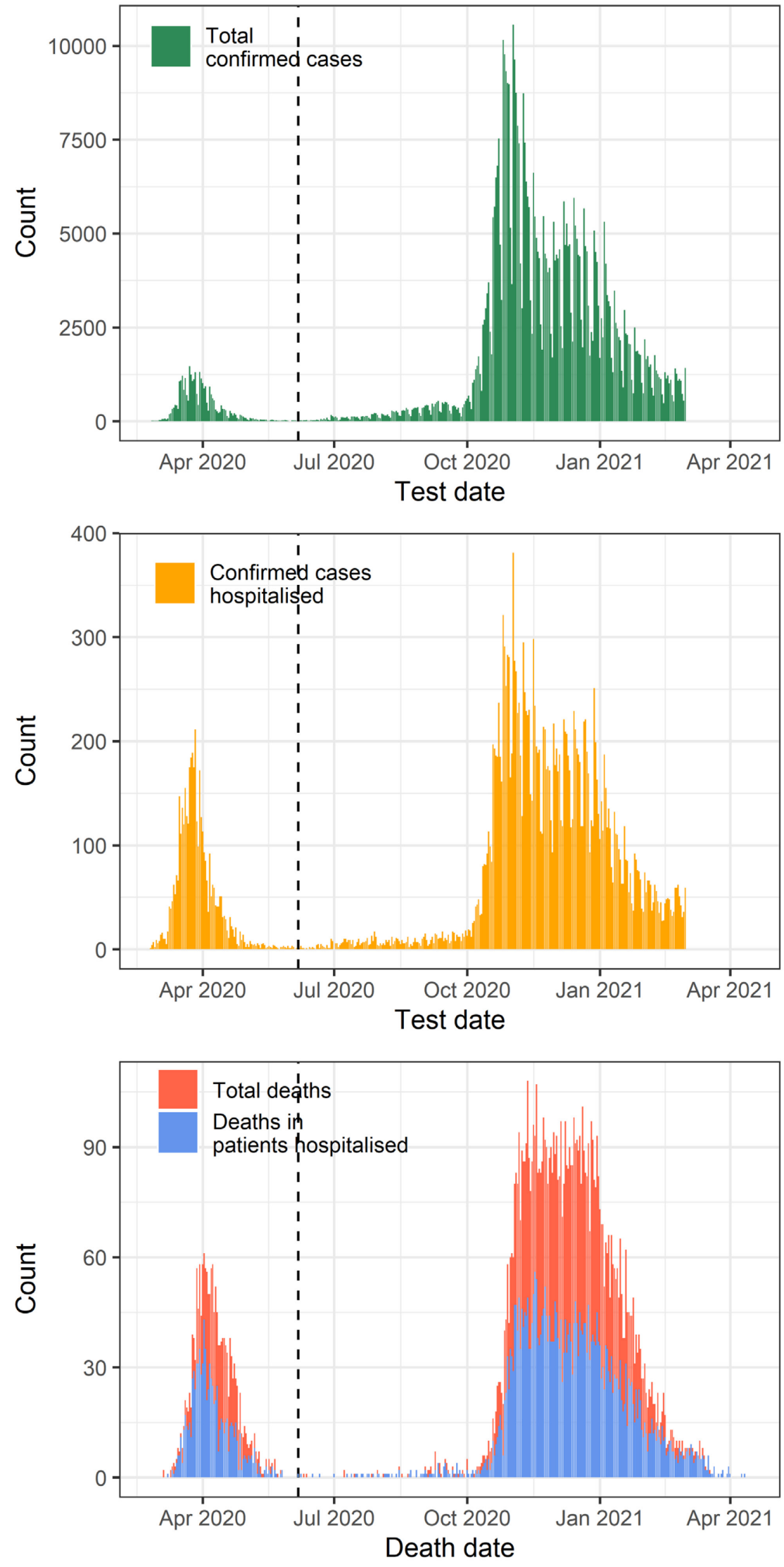


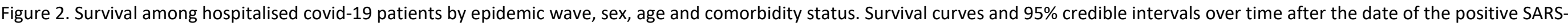

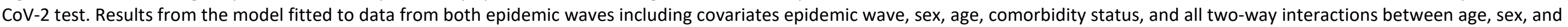
comorbidity status.

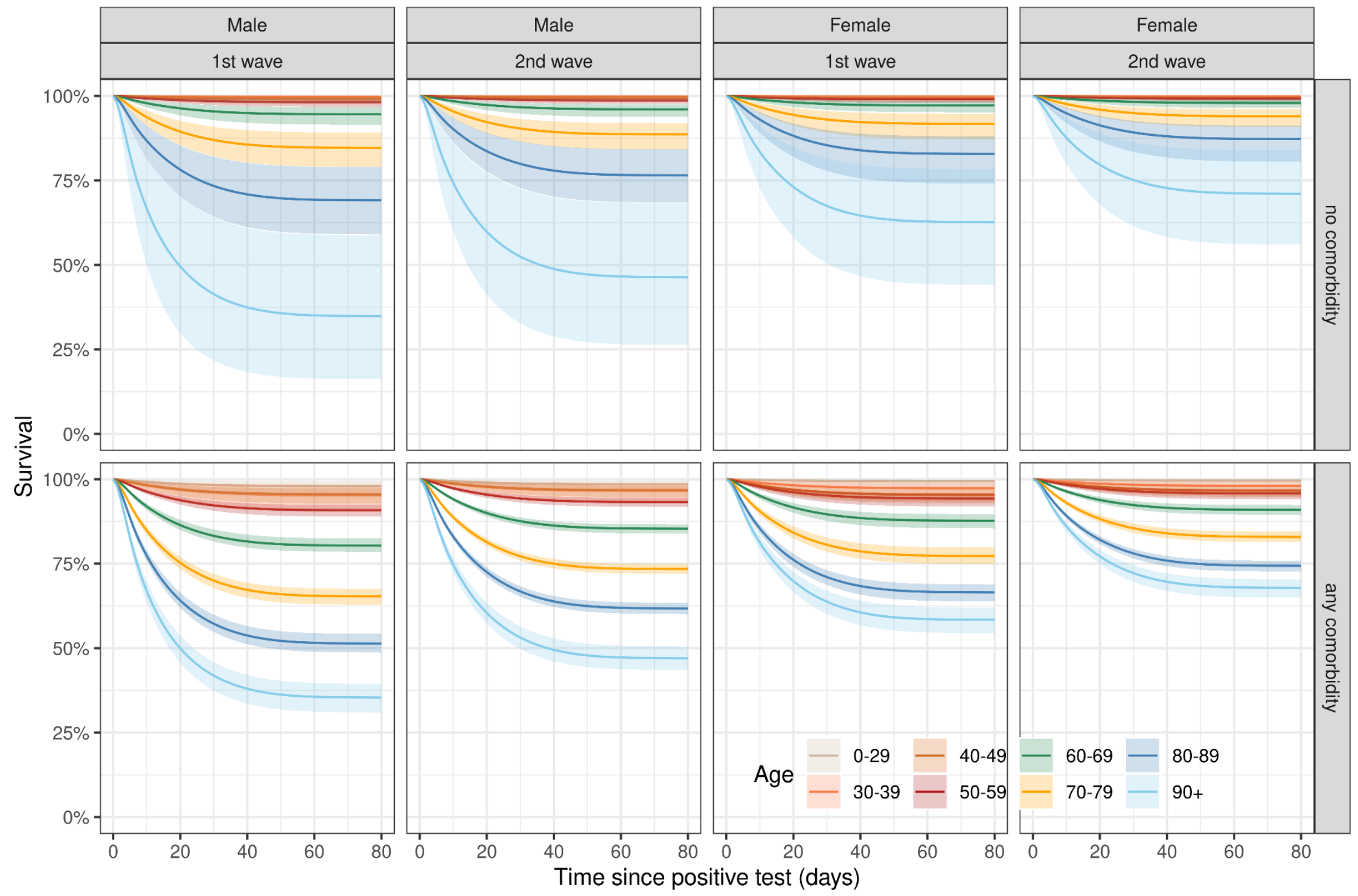




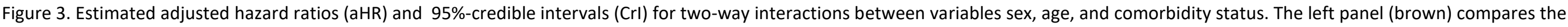

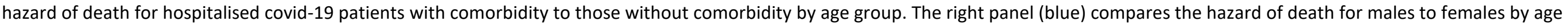

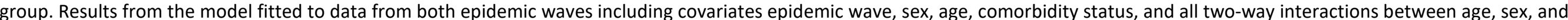
comorbidity status.
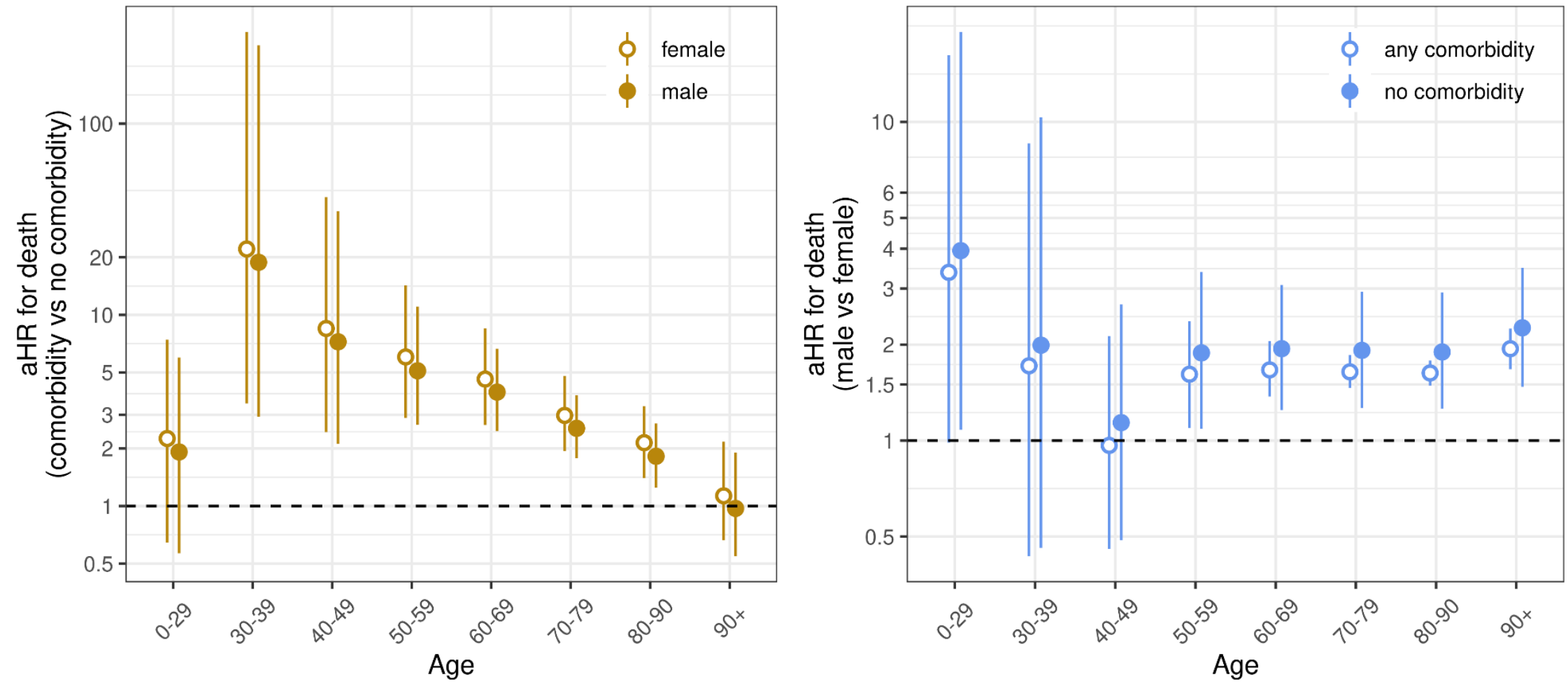
Figure 4. Survival of hospitalised covid-19 patients by ICU occupancy. Standardised predicted survival at 40 days after positive test by ICU occupancy. More transparent areas correspond to $95 \%$ credible intervals, less transparent ones to $50 \%$ credible intervals. Results from the model fitted to data from the second epidemic wave, including covariates ICU occupancy (modelled by restricted cubic splines with 3 knots), sex, age, comorbidity status, and the interaction between age and comorbidity status. Standardisation is done by averaging predictions at 40 days for the whole population by ICU occupancy in each of the posterior samples (1000 draws).

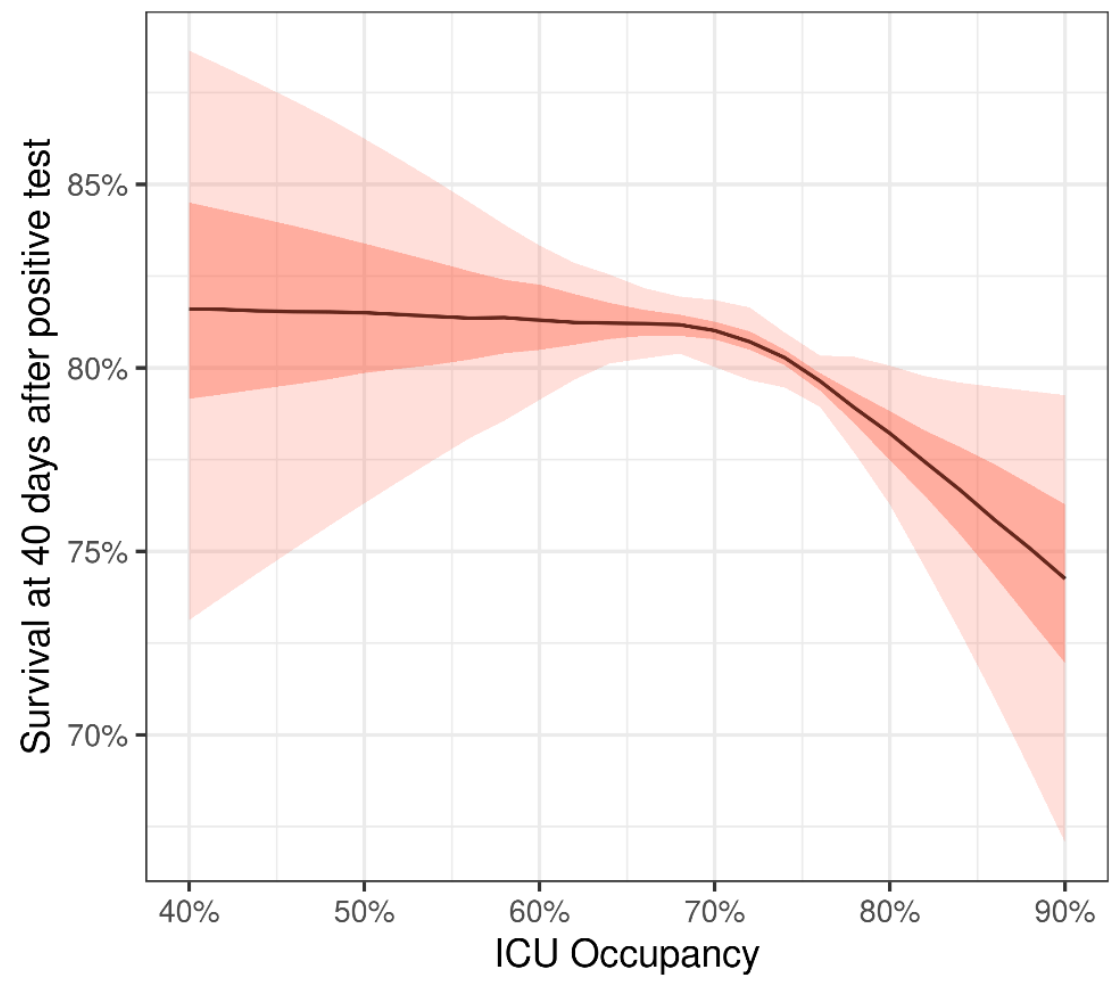




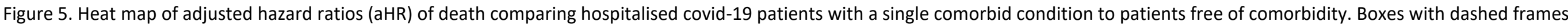

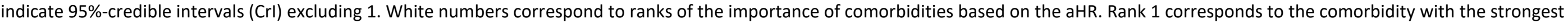

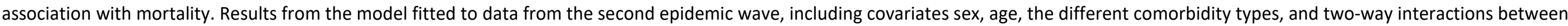
comorbidity types, between age and comorbidity types, and between sex and comorbidity types.
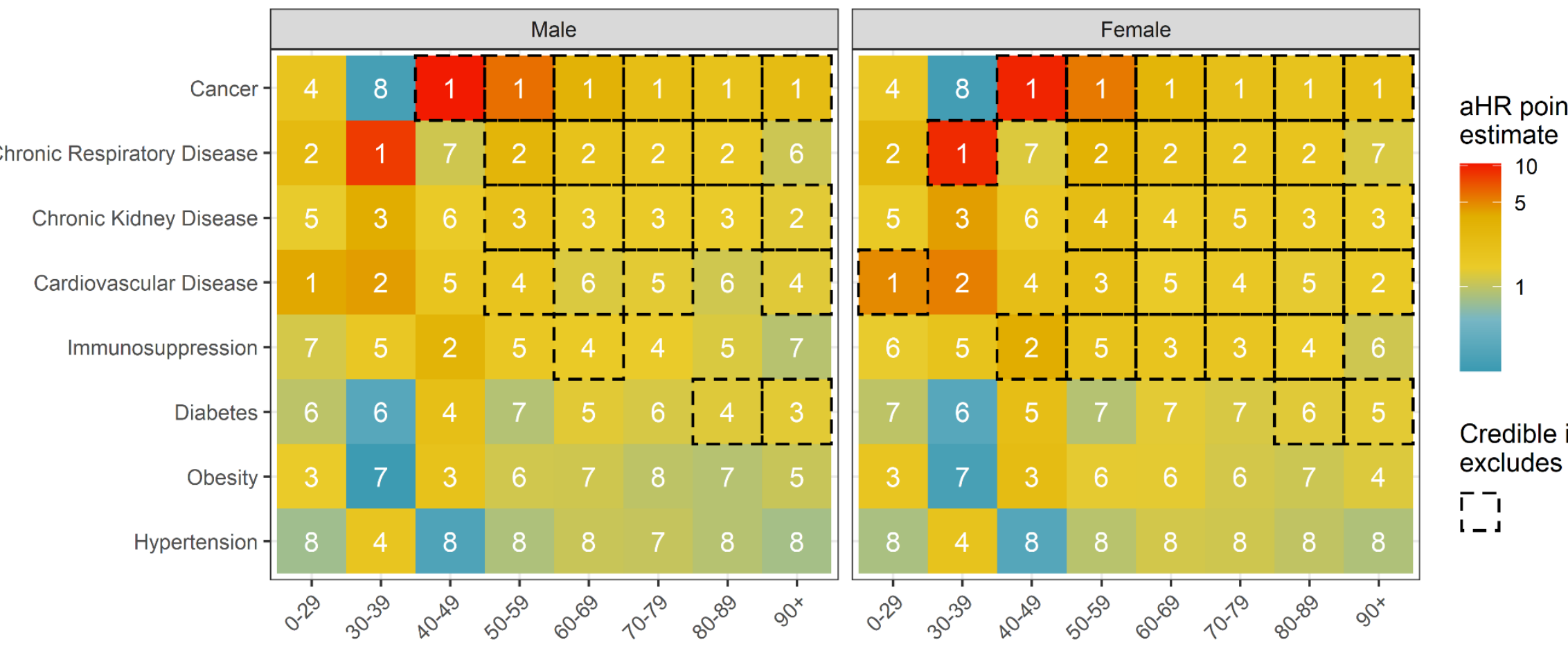

Credible interval

excludes 1.0

$i-1$

Age group 